\title{
开环葫芦脲在水中对芳烃和芳醛的增溶和对腙大环形成的促进作用
}

\author{
刘旭波林佳乐王辉张丹维* 黎占亭*
}

(复旦大学化学系 上海 200438)

\begin{abstract}
摘要 利用核磁实验, 研究了并入四个磺酸根或羧酸根的高水溶性开环葫芦脲对疏水性芳烃和芳醛的水相增溶作用. 并入四个磺酸根的开环葫芦脲 acCB-1 能显著提高包括三联苯、4,4'-二甲基联苯和联苯-4,4'-二甲醛在内的芳烃和芳醛 的水溶性, 其中 4,4'二甲基联苯的溶解度可以提高到 $8.9 \mathrm{mmol} / \mathrm{L}$, 联苯-4,4'二五醛的溶解度可以提高到 $11.2 \mathrm{mmol} / \mathrm{L}$. 并入四个羧酸根的 acCB-2 可以把五氟甲苯和六氟苯在水中的溶解度提高到 5.6 和 $3.0 \mathrm{mmol} / \mathrm{L} . \mathbf{a c C B}-1$ 还可以通过增溶 芳香醛，促进其与酰肼反应形成腙. 这一促进作用可以进一步应用于促进两个芳香二醛和一个芳香二酰肼在水中的反 应，制备常规条件下不能形成的两个芳香腙大环.
\end{abstract}

关键词 开环葫芦脲; 水相增溶; 包结; 芳烃; 芳醛; 腙; 大环化合物

\section{Water-Solubilization of Acyclic Cucurbiturils for Arenes and Aromatic Aldehydes and the Promotion for the Generation of Two Hydrazine-Based Macrocycles}

\author{
Liu, Xu-Bo \\ Lin, Jia-Le Wang, Hui Zhang, Dan-Wei* \\ Li, Zhan-Ting* \\ (Department of Chemistry, Fudan University, Shanghai 200438)
}

\begin{abstract}
The promotion of two sulfate or carboxylate-bearing acyclic cucurbiturils for the water-solubility of arenes and aromatic aldehydes is described. ${ }^{1} \mathrm{H}$ NMR experiments reveals that sulfate-bearing acyclic cucurbituril (acCB-1) signficantly improves the water-solubility of a number of arenes and aldehydes. For 4,4'-dimethylbiphenyl and biphenyl-4,4'dicarbaldehyde, the solubility can be improved to 8.9 and $11.2 \mathrm{mmol} / \mathrm{L}$, respectively. ${ }^{19} \mathrm{~F}$ NMR experiments demonstrate that carboxylate-bearing acyclic cucurbituril can increase the water-solubilities of pentafluorotoluene and hexafluorobenzene to 5.6 and $3.0 \mathrm{mmol} / \mathrm{L}$, respectively. It is also found that the water-solubilization of acCB-1 for aromatic aldehydes can promote their reaction with acylhydrazines to form hydrozone derivatives. By making use of this promotion, two hydrazone-based macrocycles can be formed from the coupling reactions of two aromatic dialdehdes and one diacylhydrazine in water.

Keywords acyclic cucurbituril; water-solubilization; encapsulation; arene; aromatic aldehyde; hydrazine; macrocycle
\end{abstract}

水是最经济和最环境友好的溶剂, 但绝大多数有机 分子在水中不溶解或溶解度很小, 因此探索发展在水中 增加有机分子溶解度的方法在理论和应用两方面都具 有价值. 利用两亲性分子形成胶束或囊泡可以实现这一 目的, 但有机分子被包埋在胶束或囊泡内部, 不与水直 接接触, 没有实现真正意义上的水相溶解 ${ }^{[1]}$. 通过大环 分子的络合或包结也可以提高有机分子在水中的溶解 性, 最典型的例子是环糊精对有机分子的包结作用 ${ }^{[2]}$. 这一增溶作用被广泛应用于药物和日用化学品研发 ${ }^{[3]}$. 离子基团修饰的柱芳烃也可以在水中通过包结作用提
高疏水性有机分子的溶解性 ${ }^{[4]}$. 葫芦腿(cucurbit $[n]$ uril, $\mathrm{CB}[n]: n$ 为重复单元数量)作为一类具有刚性疏水内穴 的大环 ${ }^{[5]}$,一定程度上类似环糊精，在水中也能包结疏 水性有机分子, 起到增溶效果和促进超分子组装 ${ }^{[6]}$. 但 葫芦脲结构修饰总体上较为困难, 并且两个应用广泛的 葫芦脲 $\mathrm{CB}[6]$ 和 $\mathrm{CB}[8]$ 水溶性很低 ${ }^{[5,7]}$, 在很大程度上限 制了获得更广泛的应用. 近年来, Isaccs 和 $\mathrm{Ma}$ 等 ${ }^{\left[{ }^{[2}-10\right]}$ 合 成了一类开环葫芦嫝 (acyclic cucurbiturils, acCB), 通过 在其端位引入多个负离子或正离子, 获得了极高的水溶 性, 并利用其包结和增溶药物, 实现了药物控制释放.

* Corresponding authors. E-mail: zhangdw@fudan.edu.cn; ztli@fudan.edu.cn

Received October 17, 2019; revised November 7, 2019; published online November 21, 2019.

Project supported by the National Natural Science Foundation of China (Nos. 21890732, 21921003).

国家自然科学基金(Nos. 21890732, 21921003)资助项目. 
进一步利用这一增溶作用, 实现一些常规条件下不能实 现的化学转换, 将有助于拓展这类分子的功能与应用. 我们利用 ${ }^{1} \mathrm{H}$ 和 ${ }^{19} \mathrm{~F}$ 核磁技术研究了分别引入四个磺酸根 和羧酸根的开环葫芦脲 acCB-1 和 acCB-2 在水中对芳 香类分子(包括含氟分子)的络合促溶作用. 在此基础上 进一步研究了 acCB-1 在水中对腙键及腙类大环形成的 促进效应, 为制备低溶解性的腙类大环化合物提供一个 新的途径.

\section{1 结果与讨论}

本项研究涉及的所有化合物的结构列于图 1. acCB$\mathbf{1}^{[9]}$ 和 $\mathbf{a c C B}-2^{[8 \mathrm{a}]}$ 根据文献方法合成. 我们主要通过核磁 测定在水中不同客体化合物的浓度, 评估两个开环葫芦 脲的增溶效果. 首先研究了芳烃化合物 $1 \sim 6$ 在浓度为 $10 \mathrm{mmol} / \mathrm{L}$ 的 acCB-1 中的增溶. 由于一些芳烃(1、2、7、 8)在盐溶液中也有一定的溶解度, 我们同时测定了这些 分子在 $20 \mathrm{mmol} / \mathrm{L}$ 的对-苯二甲酸钠(sodium terephthalate, STP)的重水溶液中的溶解度, 这一溶液具有和 acCB-1 相同的离子浓度. 我们简化处理, 把在 acCB 中 有机分子的总浓度与在 STP 中的浓度差值, 作为 acCB1 通过包结有机分子促进其溶解的判据.
化合物 acCB-1 和上述六个化合物在其溶液中达到 饱和的重水溶液的 ${ }^{1} \mathrm{H}$ NMR 列于图 2. acCB-1 与芳烃分 子形成的包结络合物可能产生不同于其游离分子的信 号, 而被包结的芳烃与游离的芳烃也可能产生两套信 号. 这些信号的化学位移与相对强度都随二者的相对浓 度和温度改变. 因此, 我们通过比较低场区(芳环上 $\mathrm{H}$ 信 号)所有信号的积分强度与 acCB-1 的 $\mathrm{OCH}_{2}$ 的积分强度, 计算出低场区 acCB-1 芳环上 $\mathrm{OCH}_{2}$ 信号强度，从而计算 出芳烃分子在低场区的积分强度，进而计算出其总溶解 度. 通过比较在 STP 溶液中的溶解度, 确定 acCB-1 通 过络合芳烃进一步提高的溶解度. 辅助材料中的图 $\mathrm{S} 1 \sim \mathrm{S} 7$ 提供了在两个溶液中记录的部分图谱. 具体结 果列于表 1 , 苯在 STP 中饱和后的核磁图谱列于辅助材 料图 S1 中. 苯(1)和对-二甲苯(2)在 STP 中有一定溶解 度 $(6.8$ 和 $2.2 \mathrm{mmol} / \mathrm{L})$, 但在 $10 \mathrm{mmol} / \mathrm{L}$ 的 $\mathbf{a c C B}-1$ 溶液 中, 溶解度增加到 29.9 和 $9.3 \mathrm{mmol} / \mathrm{L}$, 通过包结作用达 到 23.1 和 $7.1 \mathrm{mmol} / \mathrm{L}$ 的增溶效果. 联苯 $(3)$ 、三联苯 $(4)$ 、 4,4'-二甲基联苯(5)和 2,5-二溴-对-二甲苯(6)在 STP 中不 溶解，但在 acCB-1 中的溶解度分别为 2.6、0.6、1.6 和 $8.9 \mathrm{mmol} / \mathrm{L}$.
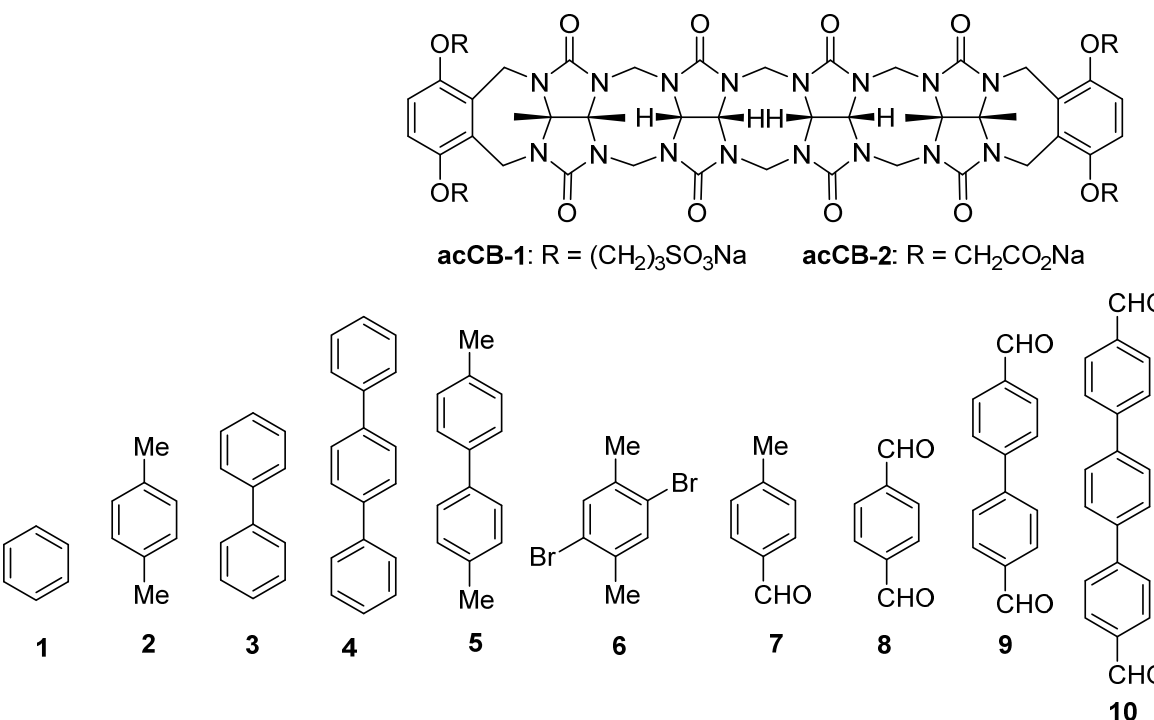<smiles>Cc1c(F)c(F)c(F)c(F)c1F</smiles><smiles>NNC(=O)CCCCC(=O)NN</smiles><smiles>[R2]Oc1cc(C(=O)N/N=C\c2cccc(C(=O)NN)c2)cc(C(=O)N/N=C/c2cccc(C=NNC(=O)c3cc([R20])cc(C(=O)NN)c3)c2)c1</smiles>

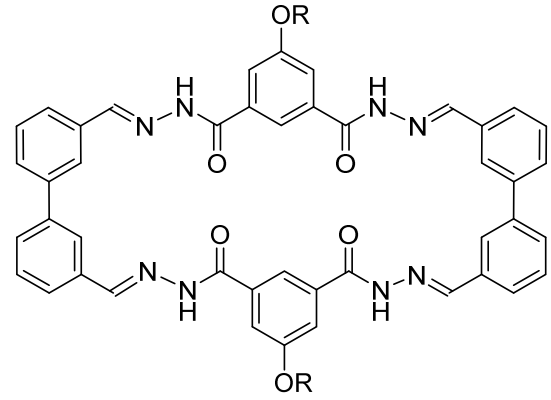

$19 \mathrm{R}=\left(\mathrm{CH}_{2} \mathrm{CH}_{2} \mathrm{O}\right)_{4} \mathrm{Me}$

图 1 化合物 $1 \sim 19$ 的结构

Figure 1 Structures of compounds $1 \sim 19$ 
表 1 开环葫芦艮 acCB-1 和对苯二甲酸钠 STP 在重水中对若干芳烃和芳醛的增溶 ${ }^{a}$

Table 1 Solubilization of acCB-1 and STP for arenes and aromatic aldehydes in $\mathrm{D}_{2} \mathrm{O}$

\begin{tabular}{|c|c|c|c|c|c|}
\hline 有机分子(G) & $\begin{array}{l}{[\mathbf{a c C B}-1] /} \\
\left(\mathrm{mmol} \cdot \mathrm{L}^{-1}\right)\end{array}$ & $\begin{array}{l}\text { acCB-1 溶液中 } \\
{[\mathbf{G}] /\left(\mathrm{mmol} \cdot \mathrm{L}^{-1}\right)}\end{array}$ & $\begin{array}{c}{[\mathrm{STP}] /} \\
\left(\mathrm{mmol} \cdot \mathrm{L}^{-1}\right)\end{array}$ & $\begin{array}{c}\text { STP 溶液中 } \\
{[\mathbf{G}] /\left(\mathrm{mmol} \cdot \mathrm{L}^{-1}\right)}\end{array}$ & $\begin{array}{c}\text { acCB-1 络合增溶浓度 } \\
\Delta[\mathbf{G}] /\left(\mathrm{mmol} \cdot \mathrm{L}^{-1}\right)\end{array}$ \\
\hline 1 & 10 & 29.9 & 20 & 6.8 & 23.1 \\
\hline 2 & 10 & 9.3 & 20 & 2.2 & 7.1 \\
\hline 3 & 10 & 2.6 & 20 & 0 & 2.6 \\
\hline 4 & 10 & 0.6 & 20 & 0 & 0.6 \\
\hline 5 & 10 & 1.6 & 20 & 0 & 1.6 \\
\hline 6 & 10 & 8.9 & 20 & 0 & 8.9 \\
\hline 7 & 10 & 19.0 & 20 & 10.0 & 9.0 \\
\hline 7 & 20 & 37.0 & 40 & 17.0 & 20.0 \\
\hline 7 & 40 & 72.0 & 80 & 32.8 & 39.2 \\
\hline 8 & 10 & 13.4 & 20 & 1.3 & 12.1 \\
\hline 8 & 20 & 16.5 & 40 & 2.0 & 14.5 \\
\hline 8 & 40 & 27.0 & 80 & 5.6 & 21.4 \\
\hline 9 & 10 & 4.0 & 20 & 0 & 4.0 \\
\hline 9 & 20 & 7.3 & 40 & 0 & 7.3 \\
\hline 9 & 40 & 11.2 & 80 & 0 & 11.2 \\
\hline 10 & 10 & 0 & 20 & 0 & 0 \\
\hline
\end{tabular}

${ }^{a}$ Determined using ${ }^{1} \mathrm{H}$ NMR $(400 \mathrm{MHz})$ in $\mathrm{D}_{2} \mathrm{O}$ at $25{ }^{\circ} \mathrm{C}$.

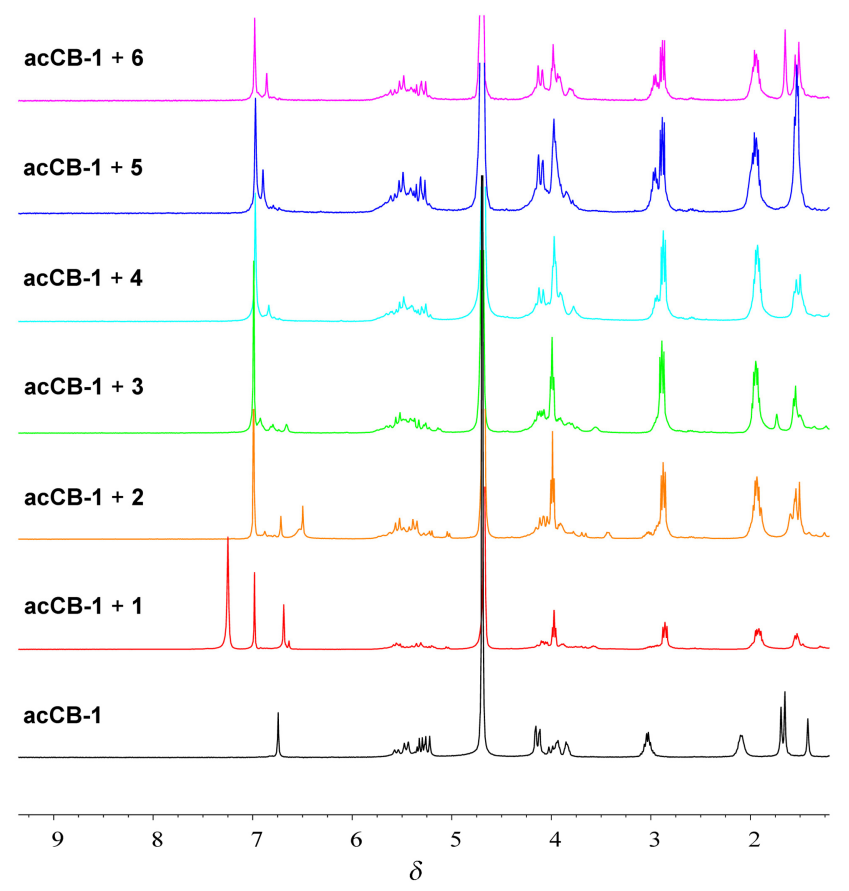

图 2 开环葫芦嫝 $\mathbf{a c C B}-1(10 \mathrm{mmol} / \mathrm{L})$ 及相应的芳烃 $\mathbf{1} \sim \mathbf{6}$ 溶 解饱和后的重水溶液的 ${ }^{1} \mathrm{H}$ NMR $(400 \mathrm{MHz})$

Figure $2{ }^{1} \mathrm{H}$ NMR $(400 \mathrm{MHz})$ of the solution of acCB-1 (10 $\mathrm{mmol} / \mathrm{L}$ ) in $\mathrm{D}_{2} \mathrm{O}$ and the related solutions that contain saturated arenes $\mathbf{1} \sim \mathbf{6}$

在上述同样测试条件下，相对于相同离子浓度的 STP, acCB-1 通过包结作用(图 3 和图 S2 S7), 对-甲基 苯甲醛 $(7)$ 、对-苯二甲醛 $(8)$ 和联苯- $4,4^{\prime}$-二甲醛 $(9)$ 的增溶 分别达到 $9.0,12.1$ 和 $4.0 \mathrm{mmol} / \mathrm{L}$. 化合物 9 在浓度为 $(10 \sim 80) \mathrm{mmol} / \mathrm{L}$ 的 STP 溶液中都不溶解. 在两个溶液 中, 三联苯-4,4"-二甲醛(10)也都没有可观察到的溶解
度, 这一化合物参照文献[12]合成. 增加 acCB-1 的浓度 可以进一步提高对化合物 7 9 的增溶效果. 例如在 40 $\mathrm{mmol} / \mathrm{L}$ 的 acCB-1 溶液中, 相对于同样离子浓度的 STP, 7 的溶解度可以进一步提高 $39.2 \mathrm{mmol} / \mathrm{L}$.

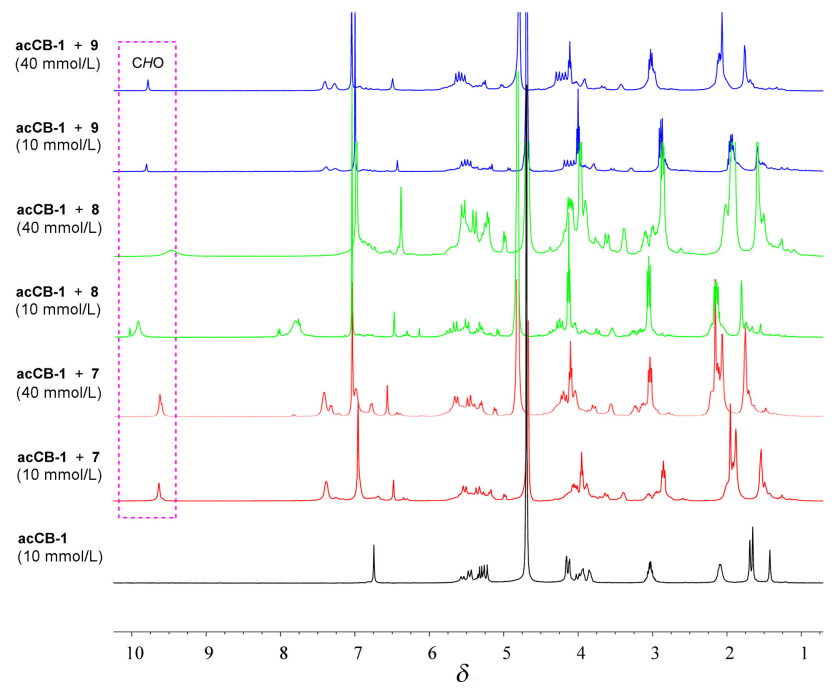

图 3 不同浓度的开环葫芦脲 acCB-1 及相应的芳烃 7 9 溶解 饱和后的重水溶液的 ${ }^{1} \mathrm{H}$ NMR $(400 \mathrm{MHz})$

Figure $3{ }^{1} \mathrm{H}$ NMR (400 MHz) of the solutions of acCB-1 of varying concentrations in $\mathrm{D}_{2} \mathrm{O}$ that contain no or saturated aromatic aldehydes $7 \sim 9$

我们又通过 ${ }^{1} \mathrm{H}$ NMR 和 ${ }^{19} \mathrm{~F} \mathrm{NMR}$ 分别研究了 $\mathbf{a c C B}-$ 2 对五氟甲苯(11)和六氟苯(12)的增溶效果. 为尽量消除 离子型化合物电离产生的影响, 我们用离子浓度相同 $(80 \mathrm{mmol} / \mathrm{L})$ 的醋酸钠溶液作为对照. 结果发现, 五氟甲 苯 11 在对照溶液中不能溶解, 但在 $20 \mathrm{mmol} / \mathrm{L}$ 的 


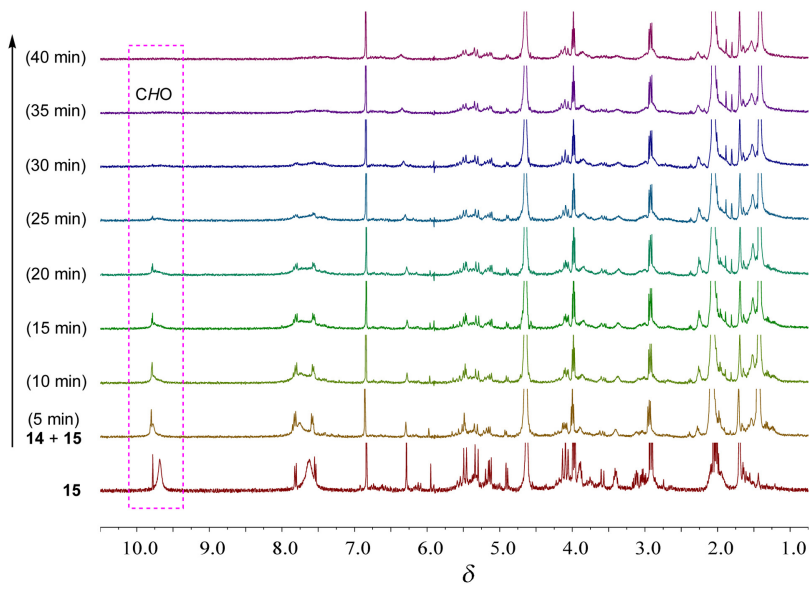

图 4 溶解有化合物 $15(2 \mathrm{mmol} / \mathrm{L})$ 的开环葫芦脲 acCB-1 (5 $\mathrm{mmol} / \mathrm{L})$ 重水溶液及加入化合物 $14(2 \mathrm{mmol} / \mathrm{L})$ 后的重水溶液 随时间变化的 ${ }^{1} \mathrm{H}$ NMR $(400 \mathrm{MHz})$

Figure $4{ }^{1} \mathrm{H}$ NMR (400 MHz) of the solution of acCB-1 (5 $\mathrm{mmol} / \mathrm{L})$ and dialdehyde $15(2 \mathrm{mmol} / \mathrm{L})$ in $\mathrm{D}_{2} \mathrm{O}$ and the same solution after addition of diacylhydrazone $14(2 \mathrm{mmol} / \mathrm{L})$ recorded at different times

acCB-2 中, 增溶的五氟甲苯受 acCB-2 屏蔽, 其 $\mathrm{CH}_{3}$ 信 号向高场移动至 $\delta 1.27$. 通过比较这一信号与 $\mathbf{a c C B}-2$ 的 $\mathrm{Ar}-\mathrm{H}$ 信号强度, 可以计算出五氟甲苯的溶解度为 5.6 $\mathrm{mmol} / \mathrm{L}$ (图 S8). 六氟苯 12 在水中即有一定的溶解度 $(1.0 \mathrm{mmol} / \mathrm{L})$. 在 $20 、 40$ 和 $80 \mathrm{mmol} / \mathrm{L}$ 的乙酸钠溶液中, 其溶解度分别为 $1.8 、 1.6$ 和 $1.1 \mathrm{mmol} / \mathrm{L}$, 即有所增加, 但 随着乙酸钠的浓度增加而降低. 但在 5、10 和 $20 \mathrm{mmol} / \mathrm{L}$ 的 acCB-2 溶液中, 其溶解度分别测定为 2.2、2.9 和 3.0 $\mathrm{mmol} / \mathrm{L}$ (图 S9), 不但总体上随 acCB-2 浓度增加而增加, 而且与乙酸钠溶液对照, 分别提高了 $0.4 、 1.3$ 和 1.9 $\mathrm{mmol} / \mathrm{L}$. 在不同浓度的 $\mathbf{a c C B}-2$ 的溶液中, ${ }^{19} \mathrm{~F} \mathrm{NMR}$ 都没 有观察到全氟联苯 12 的信号, 表明开环葫芦脲对这一 更大的有机氟化合物增溶作用有限.

基于上述实验结果, 我们进一步研究了 acCB-1 通 过增溶作用对已二酰肼 (14)和间苯二甲醛(15)及联苯3,3'-二甲醛(16)在水中反应形成酰肼/腙聚合物的可能的 促进效应. 由于三个底物在水中溶解度都很小, 并且反 应产物腙聚合物不溶于水 ${ }^{[11]}$, 这两个反应都不能在水中 顺利进行. ${ }^{1} \mathrm{H}$ NMR 揭示, 在 acCB-1 (5 mmol/L) 的重水 溶液中, 加入等摩尔量的 $(2.0 \mathrm{mmol} / \mathrm{L}) 14$ 到增溶的二醛 $15(2.0 \mathrm{mmol} / \mathrm{L})$ 的相应溶液中, 反应能快速进行, 大约 $30 \mathrm{~min}$ 后, 化学位移在 $\delta 9.4$ 周围的醛基 $\mathrm{CHO}$ 特征信号 完全消失(图 4). 当 acCB-1 浓度提高 $20 \mathrm{mmol} / \mathrm{L}$ 时, 反 应在约 $10 \mathrm{~min}$ 后即能完成. 尽管由于产物成分复杂, 不 能进一步研究这一反应的产物选择性, 这些结果证明, acCB-1 可以通过促溶作用促进酰肼和醛形成腙键. 同 样地, 在 $20 \mathrm{mmol} / \mathrm{L}$ 浓度的 acCB-1 重水溶液中, 14 和 16 反应形成腙衍生物的反应, 在约 $20 \mathrm{~min}$ 内即可完成
(图 S10).

由于 acCB-1 能显著促进酰肼和醛在水中缩合形成 腙，我们又根据文献[13]合成了化合物 17. 尽管引入一 个亲水的乙氧基链, 这一化合物在水中的溶解度仍然很 低, 和溶解度同样较低的等摩尔量的 15 或 16 在水中反 应， ${ }^{1} \mathrm{H}$ NMR 表明有反应发生，但形成不能分离的沉淀， 其最有可能是不同长度的寡聚腙链. 两个反应的底物都 不能完全转化. 在 $5 \mathrm{mmol} / \mathrm{L}$ 的 $\mathbf{a c C B}-1$ 水溶液中, 二醛 15 和 16 可以增溶到 2.0 和 $1.1 \mathrm{mmol} / \mathrm{L}$. 在这两个溶液中 分批加入等摩尔量的 17 , 其与两个二醛反应, 形成黄色 沉淀. 在乙腈和二甲基亚砜中重结晶，可以得到相应 2 +2 大环 18 和 19, 产率在 $16 \%$ 和 $12 \%$. 尽管两个大环化 合物的产率仍然较低, 但由于在纯水中不能形成这些大 环, acCB-1 对底物的增溶作用对于两个大环的形成起到 重要促进作用.

\section{2 结论}

通过核磁实验揭示, 高水溶性的开环葫芦嫝能够通 过包结作用显著提高疏水性芳烃、芳香醛和氟代芳烃的 水溶性. 这一水相增溶作用可以促进芳香醛与酰肼在水 中缩合形成腙类分子, 并进一步应用于合成具有低溶解 性能的腙类大环. 由芳香单体形成的腙类大环分子, 由 于共轭大环的强堆积作用, 其溶解性一般很低, 需要引 入多个长的亲水侧链或正离子才能制备, 分离和纯化相 应大环 ${ }^{[14]}$. 本文研究结果显示, 通过开环葫芦脲的水相 增溶作用, 可以实现普通条件下不能进行的一些反应, 为一些低溶解性的特殊结构分子特别是大环分子的合 成提供了一个新的思路 ${ }^{[15]}$.

\section{3 实验部分}

\section{1 仪器和试剂}

溶剂和试剂使用前没有进一步纯化. 核磁在 $400 \mathrm{M}$ Bruker Avance III HD 型核磁共振仪记录, 质谱在 Bruker McriOTOF 11 型质谱仪测试. $\mathbf{a c C B}-1^{[9 a]}$, acCB-2 ${ }^{[8 a]}$, $10^{[12]}$ 和 $17^{[13]}$ 根据文献方法合成, 其它化合物为商品试 剂. 核磁实验中, 内标对苯二甲酸钠 $(\mathrm{STP})$ 的浓度直接 通过称重样品确定，被增溶分子的浓度通过比较其相应 的探针信号和 STP 的 $\mathrm{CH}$ 信号相对积分计算。

\section{2 化合物 18 的合成}

在化合物 acCB-1 (0.15 g, $0.10 \mathrm{mmol})$ 的水溶液中 $(20 \mathrm{~mL})$ 加入化合物 15 (5.4 mg, $0.04 \mathrm{mmol})$. 室温搅拌 $10 \mathrm{~min}$ 后, 向溶液中加入化合物 17 (16 mg, $0.04 \mathrm{mmol})$. 继续搅拌 $1 \mathrm{~h}$ 后, 形成的黄色沉淀离心分离, 用甲醇和 乙醚分别洗涤后, 离心分离, 之后在乙腈和二甲基亚砜 $(V: V=3: 1)$ 中重结晶, 得到化合物 18 (6.4 mg, 16\%)为 
黄色固体. m.p. $>250{ }^{\circ} \mathrm{C}$ (dec.); ${ }^{1} \mathrm{H}$ NMR (DMSO- $\left.d_{6}\right) \delta$ : $12.01(\mathrm{~s}, 4 \mathrm{H}, \mathrm{NH}), 8.54(\mathrm{~s}, 4 \mathrm{H}), 8.18 \sim 8.13(\mathrm{br}, 2 \mathrm{H})$, $8.09 \sim 8.05$ (br, $2 \mathrm{H}), 7.80 \sim 7.75(\mathrm{br}, 4 \mathrm{H}), 7.68 \sim 7.65$ (br, $4 \mathrm{H}), 7.58 \sim 7.54(\mathrm{br}, 2 \mathrm{H}), 4.29 \sim 4.25(\mathrm{~m}, 4 \mathrm{H}), 3.83 \sim 3.80$ $(\mathrm{m}, 4 \mathrm{H}), 3.68 \sim 3.10(\mathrm{~m}, 30 \mathrm{H})$. 由于分辨率较低, 该 ${ }^{1} \mathrm{H}$ $\mathrm{NMR}$ 芳环上 $\mathrm{H}$ 没有出现预期的信号裂分. ${ }^{13} \mathrm{C} \mathrm{NMR}$ (DMSO- $\left.d_{6}\right) \delta$ : 174.1, 162.7, 148.4, 145.8, 140.6, 135.4, 134.1, 130.0, 129.0, 125.6, 71.6, 70.3, 70.2, 69.9, 69.2, 68.1, 67.9, 58.3; HRMS (MALDI-TOF) calcd for $\mathrm{C}_{50} \mathrm{H}_{60^{-}}$ $\mathrm{N}_{8} \mathrm{O}_{14}$ 996.4229, found 996.4241.

\section{3 化合物 19 的合成}

参照上述方法, 该化合物由 16 和 17 反应制备, 为 黄色固体, 产率 $12 \%$. m.p. $>275{ }^{\circ} \mathrm{C}$ (dec.); ${ }^{1} \mathrm{H}$ NMR $\left(\mathrm{DMSO}-d_{6}\right) \delta: 12.25(\mathrm{~s}, 4 \mathrm{H}), 8.70(\mathrm{~s}, 8 \mathrm{H}), 8.35(\mathrm{~s}, 2 \mathrm{H})$, $8.11(\mathrm{~s}, 4 \mathrm{H}), 7.87 \sim 7.83(\mathrm{~m}, 4 \mathrm{H}), 7.74(\mathrm{~s}, 4 \mathrm{H}), 7.64 \sim 7.60$ $(\mathrm{m}, 4 \mathrm{H}), 4.30 \sim 4.26(\mathrm{~m}, 4 \mathrm{H}), 3.85 \sim 3.80(\mathrm{~m}, 4 \mathrm{H}), 3.69 \sim$ $3.11(\mathrm{~m}, 30 \mathrm{H})$. 由于分辨率较低, 该 ${ }^{1} \mathrm{H}$ NMR 芳环上 $\mathrm{H}$ 没有出现预期的信号裂分. ${ }^{13} \mathrm{C} \mathrm{NMR}\left(\mathrm{DMSO}-d_{6}\right) \delta$ : $193.7,162.8,159.0,148.4,140.7,135.5,133.3,129.2$, 127.3, 125.9, 120.0, 117.2, 71.4, 70.5, 70.3, 70.1, 70.0, 69.3, 68.3, 58.5; HRMS (MALDI-TOF) calcd for $\mathrm{C}_{62} \mathrm{H}_{68}-$ $\mathrm{N}_{8} \mathrm{O}_{14} 1148.4855$, found 1148.4831 .

辅助材料 (Supporting Information) 对苯二甲酸钠 (STP) $(20 \mathrm{mmol} / \mathrm{L})$ 和苯 $(\mathbf{1})$ 饱和的STP的重水溶液的 ${ }^{1} \mathrm{H}$ NMR, 对甲基苯甲醛(7)饱和的开环葫芦脲 acCB-1 (20 $\mathrm{mmol} / \mathrm{L})$ 的重水溶液的 ${ }^{1} \mathrm{H} \mathrm{NMR}$, 对苯二甲醛 $(8)$ 饱和的 开环葫芦脲 acCB-1 $(20 \mathrm{mmol} / \mathrm{L})$ 的重水溶液的 ${ }^{1} \mathrm{H}$ NMR, 联苯 -4,4'-二甲醛 (9) 饱和的开环葫芦嫝 acCB-1 (20 $\mathrm{mmol} / \mathrm{L})$ 的重水溶液的 ${ }^{1} \mathrm{H} \mathrm{NMR}$, 对苯二甲醛 $(8)$ 饱和的 对苯二甲酸钠 $(20 \mathrm{mmol} / \mathrm{L})$ 的重水溶液的 ${ }^{1} \mathrm{H} \mathrm{NMR}$, 对苯 二甲醛 $(8)$ 饱和的对苯二甲酸钠 $(80 \mathrm{mmol} / \mathrm{L})$ 的重水溶液 的 ${ }^{1} \mathrm{H} \mathrm{NMR}$, 联苯-4,4'-二甲醛(9)饱和的对-苯二甲酸钠 $(20 \mathrm{mmol} / \mathrm{L})$ 的重水溶液的 ${ }^{1} \mathrm{H} \mathrm{NMR}$, 五氟甲苯 $(\mathbf{1 1})$ 饱和 的开环葫芦嫝 acCB-1 (20 mmol/L) 和三氟乙酸钠 $(10$ $\mathrm{mmol} / \mathrm{L})$ 重水溶液的 ${ }^{1} \mathrm{H} \mathrm{NMR}$, 开环葫芦脲 $(20 \mathrm{mmol} / \mathrm{L})$ 重水溶液的 ${ }^{1} \mathrm{H}$ NMR, 五氟甲苯饱和 $(11)$ 的乙酸钠 $(80$ $\mathrm{mmol} / \mathrm{L})$ 和三氟乙酸钠 $(10 \mathrm{mmol} / \mathrm{L})$ 重水溶液的的 ${ }^{1} \mathrm{H}$ NMR. 溶解有化合物 16 (2 mmol/L) 的开环葫芦嫝 acCB-1 (5 mmol/L) 重水溶液的 ${ }^{1} \mathrm{H}$ NMR及加入化合物 14 $(2 \mathrm{mmol} / \mathrm{L})$ 后的重水溶液随时间变化的 ${ }^{1} \mathrm{H} \mathrm{NMR}$, 化合 物 19在DMSO- $d_{6}$ 中的 ${ }^{1} \mathrm{H}$ NMR. 化合物 18 和 19 在DMSO$d_{6}$ 中的 ${ }^{13} \mathrm{C} \mathrm{NMR}$. 六氟苯(12)饱和的开环葫芦脲 $\mathbf{a c C B - 1}$ $(20 \mathrm{mmol} / \mathrm{L})$ 和三氟乙酸钠 $(10 \mathrm{mmol} / \mathrm{L})$ 重水溶液的 ${ }^{19} \mathrm{~F}$ $\mathrm{NMR}$, 六氟苯饱和的乙酸钠 $(80 \mathrm{mmol} / \mathrm{L})$ 和三氟乙酸钠
$(10 \mathrm{mmol} / \mathrm{L})$ 重水溶液的 ${ }^{19} \mathrm{~F} \mathrm{NMR}$, 六氟苯饱和的三氟乙 酸钠 $(10 \mathrm{mmol} / \mathrm{L})$ 重水溶液的 ${ }^{19} \mathrm{~F} \mathrm{NMR}$, 六氟苯饱和的重 水溶液的 ${ }^{19} \mathrm{~F}$ NMR图谱. 这些材料可以免费从本刊网站 (http://sioc-journal.cn/)上下载.

\section{References}

[1] (a) Birch, H.; Redman, A. D.; Letinski, D. J.; Lyon, D. Y.; Mayer, P. Anal. Chim. Acta 2019, 1086, 16.

(b) Li, H.; Jia, X.; Li, Y.; Shi, X.; Hao, J. J. Phys. Chem. B 2006, $110,68$.

(c) Chen, Y.; Huang, F.; Li, Z.-T.; Liu, Y. Sci. China Chem. 2018, 61,979 .

(d) Yang, Z.; Peng, Y.; Qiu, L. Chin. Chem. Lett. 2018, 29, 1839.

(e) Wang, K.; Guo, D.-S.; Liu, Y. Chem.-Eur. J. 2012, 18, 8758.

(f) Wang, K.; Guo, D.-S.; Liu, Y. Chem.-Eur. J. 2010, 16, 8006.

[2] (a) Liu, R.; Zhang, Y.; Wu, W.; Liang, W.; Huang, Q.; Yu, X.; Xu, W.; Zhou, D.; Selvapalam, N.; Yang, C. Chin. Chem. Lett. 2019, 30, 577 .

(b) Zhang, L.; Zhang, Y.-M.; Liu, G.; Liu, Y. Chin. Chem. Lett. 2019, 30, 120 .

(c) Zhang, Y.-M.; Xu, Q.-Y.; Liu, Y. Sci. China Chem. 2019, 62, 549 .

(d) Xu, C.; Xu, L.; Ma, X. Chin. Chem. Lett. 2018, 29, 970.

(e) Zhao, Q.; Chen, Y.; Liu, Y. Chin. Chem. Lett. 2018, 29, 84.

(f) Song, S.; Li, M.; Gong, X.; Han, H.; Zhou, Y.; Wang, L.; Shuang, S.; Dong, C. Chem. Res. Chin. Univ. 2018, 34, 203.

(g) Zhang, Y.; Chen, Y.; Li, J.; Liang, L.; Liu, Y. Acta Chim. Sinica 2018, 76, 622 (in Chinese).

(张依, 陈涌, 李晶晶, 梁璐, 刘育, 化学学报, 2018, 76, 622.)

(h) Wang, R.; Wei, Z.; Guo, J.; Feng, Y.; Xu, E.; Duan, H.; Lin, Y.; Yang, Q.; Du, J.; Li, Y. Chem. Res. Chin. Univ. 2018, 34, 180.

(i) Chen, X.-M.; Chen, Y.; Liu, Y. Chin. J. Chem. 2018, 36, 526.

(j) Liu, A.; Xiong, C.; Ma, X.; Ma, W.; Sun, R. Chin. J. Chem. 2019, 37, 793 .

(k) Song, C.; Xiao, Y.; Li, K.; Zhang, X.; Lu, Y. Chin. Chem. Lett. 2019, 30, 1249.

(1) Fang, X.; Yan, D. Sci. China Chem. 2018, 61, 397.

[3] (a) Loftsson, T.; Brewster, M. E. J. Pharm. Sci. 1996, 85, 1017. (b) Uekaji, Y.; Terao, K. J. Inclusion Phenom. Macrocyclic Chem.. 2019, 93, 3 .

(c) Shao, W.; Liu, X.; Wang, T.; Hu, X. Chin. J. Org. Chem. 2018, 38, 1107 (in Chinese).

(邵为, 刘昕, 王婷婷, 胡晓玉, 有机化学, 2018, 38, 1107.)

(d) Li, J.; Pan, D.; Yi, J.; Hao, L.; Kang, Q.; Liu, X.; Lu, L.; Lu, J. Carbohydr. Polym. 2019, 223, 115115.

(e) Liu, G.; Yuan, Q.; Hollett, G.; Zhao, W.; Kang, Y.; Wu, J. Polym. Chem. 2018, 9, 3436.

(f) Bian, X.; Zhao, B.; Pang, B.; Zheng, Z.; Liu, S.; Liu, Z.; Song, F. Chin. J. Chem. 2019, 37, 581.

(g) Yang, Z.; Peng, Y.; Qiu, L. Chin. Chem. Lett. 2018, 29, 1839.

(h) Liang, L.; Chen, Y.; Chen, X.-M.; Zhang, Y.; Liu, Y. Chin. Chem. Lett. 2018, 29, 989.

(i) Wang, X.; Yang, Y.; Fan, L.; Yang, F.; Wu, D. Sci. China Chem. 2018, 61, 311.

[4] (a) Ma, Y.; Xue, M.; Zhang, Z.; Chi, X.; Huang, F. Tetrahedron 2013, 69,4532 .

(b) Li, Z.; Hou, N.; Shao, W.; Xiao, S.; Lin, C.; Wang, L. Chin. J. Org. Chem. 2018, 38, 2002 (in Chinese).

(李臻益, 候娜娜, 邵为, 肖守军, 林晨, 王乐勇, 有机化学, 2018, 38, 2002.)

(c) Shu, X.; Xu, K.; Hou, D.; Li, C. Isr. J. Chem. 2018, 58, 1194.

(d) Li, Z.; Yang, J.; Huang, F. Chin. J. Chem. 2018, 36, 59.

(e) Yang, X.; Mao, W.; Liu, Y.; Li, L.; Ma, D. Chin. J. Chem. 2019, 37,575 .

[5] (a) Mock, W. L. Top. Curr. Chem. 1995, 175, 1. 
(b) Lagona, J.; Mukhopadhyay, P.; Chakrabarti, S.; Isaacs, L. Angew. Chem., Int. Ed. 2005, 44, 4844.

(c) Yang, X.; Liu, F.; Zhao, Z.; Liang, F.; Zhang, H.; Liu, S. Chin. Chem. Lett. 2018, 29, 1560.

(d) Jiao, Y.; Zhang, X. Acta Chim. Sinica 2018, 76, 659 (in Chinese).

(焦阳, 张希, 化学学报, 2018, 76, 659.)

(e) Wang, H.; Kan, J.; Bian, B.; Chen, Q.; Tao, Z.; Xiao, X. Chin. J. Org. Chem. 2018, 38, 3094 (in Chinese).

(王海燕, 阚京兰, 边炳, 陈青, 陶朱, 肖昕, 有机化学, 2018, 38, 3094.)

(f) Yu, S.-B.; Qi, Q.; Yang, B.; Wang, H.; Zhang, D.-W.; Liu, Y.; Li, Z.-T. Small 2018, 14, 1801037.

(g) Li, X.-F.; Yu, S.-B.; Yang, B.; Tian, J.; Wang, H.; Zhang, D.-W.; Liu, Y.; Li, Z.-T. Sci. China Chem. 2018, 61, 830

(h) Zhang, B.; Dong, Y.; Li, J.; Yu, Y.; Li, C.; Cao, L. Chin. J. Chem. 2019, 37, 269

(i) Bai, D.; Zhou, Y.; Lu, J.; Liu, Q.; Chen, Q.; Tao, Z.; Xiao, X. Chin. J. Org. Chem. 2018, 38, 1477 (in Chinese).

(白东, 周杨, 卢季红, 刘青云, 陈青, 陶朱, 肖昕, 有机化学, 2018, 38, 1477.)

(j) Xu, H.; Wang, Q. Chin. Chem. Lett. 2019, 30, 337.

(k) Wu, W.; Song, S.; Cui, X.; Sun, T.; Zhang, J.-X.; Ni, X.-L. Chin. Chem. Lett. 2018, 29, 95.

(1) Wu, Y.-P.; Yan, M.; Gao, Z.-Z; Hou, J.-L.; Wang, H.; Zhang, D.-W.; Zhang, J.; Li, Z.-T. Chin. Chem. Lett. 2019, 30, 1383.

(m) Yan, M.; Liu, X.-B.; Gao, Z.-Z.; Wu, Y.-P.; Hou, J.-L.; Wang, H.; Zhang, D.-W.; Liu, Y.; Li, Z.-T. Org. Chem. Front. 2019, 6, 1698.

(n) Wu, Y.-P.; Wang, Z.-K.; Wang, H.; Zhang, D.-W.; Zhao, X.; Li, Z.-T. Acta Chim. Sinica 2019, 77, 735 (in Chinese).

(吴义鹏, 王泽坤, 王辉, 张丹维, 赵新, 黎占亭, 化学学报, 2019, 77, 735.)

(o) Li, T.-T.; Wen, L.-L.; Ji, H.-L.; Liu, F.-Y.; Sun, S.-G. Chin. Chem. Lett. 2017, 28, 463.

[6] (a) Huang, J.; Xu, Z.; Lian, X.; Zhang, X.; Tao, Z.; Zhou, Q.; Zhang, Q.; Wei, G. Chem. J. Chin. Univ. 2018, 39, 2425 (in Chinese).

(黄净净, 许志玲, 练小卫, 张晓东, 陶朱, 周清娣, 张前军, 卫 钢, 高等学校化学学报, 2018, 39, 2425.)

(b) Lian, X.; Huang, J.; Tao, Z.; Zhou, Q.; Zhang, Q.; Wei, G. Chem. J. Chin. Univ. 2018, 39, 226 (in Chinese). (练小卫, 黄净净, 陶朱, 周清娣, 张前军, 卫钢, 高等学校化学 学报, 2018, 39, 226.)

(c) Li, S.; Chan, J. Y.-W.; Li, Y.; Bardelang, D.; Zheng, J.; Yew, W. W.; Chan, D. P.-C.; Lee, S. M. Y.; Wang, R. Org. Biomol. Chem. 2016, 14,7563

(d) Chu, J. J.; Apps, M. G.; Wheate, N. J. Supramol. Chem. 2014, 26, 648

(e) Ma, W.-J.; Chen, J.-M.; Jiang, L.; Yao, J.; Lu, T.-B. Mol. Pharm. 2013, 10, 4698 .

(f) Huang, Y.; Xue, S.-F.; Tao, Z.; Zhu, Q.-J.; Zhang, H.; Lin, J.-X.;
Yu, D.-H. J. Inclusion Phenom. Macrocyclic Chem. 2008, 61, 171. (g) Zhao, Y.; Buck, D. P.; Morris, D. L.; Pourgholami, M. H.; Day, A. I.; Collins, J. G. Org. Biomol. Chem. 2008, 6, 4509.

(h) Yang, B.; Zhang, X.-D.; Li, J.; Tian, J.; Wu, Y.-P.; Yu, F.-X.; Wang, R.; Wang, H.; Zhang, D.-W.; Liu, Y.; Zhou, L.; Li, Z.-T. CCS Chem. 2019, 1, 156.

(i) Zhang, Z.; Kan, J.; Feng, H.; Liu, Q.; Tao, Z.; Xiao, X. Chin. J. Org. Chem. 2018, 38, 1972 (in Chinese).

(张智睿，阚京兰，冯华明，刘青云，陶朱，肖昕，有机化学， 2018, 38, 1972.)

(j) Zhang, T.; Liu, Y.; Hu, B.; Zhang, C.; Chen, Y.; Liu, Y. Chin. Chem. Lett. 2019, 30, 949.

(k) Yi, J.; Song, S.; Zhang, S.; Zhang, S.; Tian, M.; Ni, X. Chem. J. Chin. Univ. 2018, 39, 911 (in Chinese).

(易君明，宋森，张胜，张少威，田蒙奎，倪新龙，高等学校化学 学报, 2018, 39, 911.)

(1) Bai, D.; Wang, X.; Gao, Z.; Qiu, S.; Tao, Z.; Zhang, J.; Xiao, X Chin. J. Org. Chem. 2017, 37, 2022 (in Chinese)

(白东, 王金鍂, 高中政, 邱胜超, 陶朱, 张建新, 肖昕, 有机化学, 2017, 37, 2022.)

(m) Yin, Z.-J.; Wu, Z.-Q.; Lin, F.; Qi, Q.-Y.; Xu, X.-N.; Zhao, X. Chin. Chem. Lett. 2017, 28, 1167.

(n) Gao, Z.; Yang, L.; Bai, D.; Chen, L.; Tao, Z.; Xiao, X. Chem. J. Chin. Univ. 2017, 38, 212 (in Chinese).

(高中政, 杨立国, 白东, 陈丽霞, 陶朱, 肖昕, 高等学校化学学 报, 2017, 38, 212.)

[7] Lee, J. W.; Samal, S.; Selvapalam, N.; Kim, H.-J.; Kim, K. Acc. Chem. Res. 2003, 36, 621.

[8] (a) Ma, D.; Zavalij, P. Y.; Isaacs, L. J. Org. Chem. 2010, 75, 4786.

(b) Ma, D.; Glassenberg, R.; Ghosh, S.; Zavalij, P. Y.; Isaacs, L. Supramol. Chem. 2012, 24, 325.

(c) Zhang, M.; Sigwalt, D.; Isaacs, L. Chem. Commun. 2015, 51, 14620 .

[9] (a) Ma, D.; Hettiarachchi, G.; Nguyen, D.; Zhang, B.; Wittenberg, J. B.; Zavalij, P. Y.; Briken, V.; Isaacs, L. Nat. Chem. 2012, 4, 503 (b) Zhang, B.; Zavalij, P. Y.; Isaacs, L. Org. Biomol. Chem. 2014 $12,2413$.

[10] (a) Mao, D.; Liang, Y.; Liu, Y.; Zhou, X.; Ma, J.; Jiang, B.; Liu, J.; Ma, D. Angew. Chem., Int. Ed. 2017, 56, 12614.

(b) Mao, W.; Mao, D.; Yang, F.; Ma, D. Chem.-Eur. J. 2019, 25, 2272 .

[11] Choi, H.; Ahn, J.; Kim, S.; Kim, H.; Jung, J. H. Chem. Commun. 2016, 52, 7600 .

[12] Kotha, S.; Shah, V. R. Synthesis 2007, 3653.

[13] Zha, D.; You, L. ACS Appl. Mater. Interfaces 2016, 8, 2399.

[14] Zhang, D.; Li, Z. Chin. J. Org. Chem. 2012, 32, 2009 (in Chinese). (张丹维, 黎占亭, 有机化学, 2012, 32, 2009.)

[15] Wei, X.; Gu, J.; Liu, X.; Huang, C.; Zhu, B. Chin. J. Org. Chem. 2018, 38, 3386 (in Chinese).

(魏小康, 谷静池, 刘兴丽, 黄超, 朱必学, 有机化学, 2018, 38, 3386.)

(Cheng, F.) 